Proceedings of the Edinburgh Mathematical Society (2007) 50, 229-249 (C)

DOI:10.1017/S0013091504001233 Printed in the United Kingdom

\title{
ALMOST-PERIODIC SOLUTIONS FOR AN ECOLOGICAL MODEL WITH INFINITE DELAYS
}

\author{
YONGHUI XIA ${ }^{1}$ AND JINDE CAO ${ }^{2}$ \\ ${ }^{1}$ College of Mathematics and Computer Science, Fuzhou University, Fuzhou 350002, \\ People's Republic of China (yhxia@fzu.edu.cn) \\ ${ }^{2}$ Department of Mathematics, Southeast University, Nanjing 210096, \\ People's Republic of China (jdcao@seu.edu.cn)
}

(Received 6 November 2004)

Abstract By using Lebesgue's dominated convergence theorem and constructing a suitable Lyapunov functional, we study the following almost-periodic Lotka-Volterra model with $M$ predators and $N$ prey of the integro-differential equations

$$
\begin{aligned}
\dot{x}_{i}(t)=x_{i}(t)\left[b_{i}(t)-a_{i i}(t) x_{i}(t)-\right. & \sum_{k=1, k \neq i}^{N} a_{i k}(t) \int_{-\infty}^{t} H_{i k}(t-\sigma) x_{k}(\sigma) \mathrm{d} \sigma \\
& \left.-\sum_{l=1}^{M} c_{i l}(t) \int_{-\infty}^{t} K_{i l}(t-\sigma) y_{l}(\sigma) \mathrm{d} \sigma\right], \quad i=1,2, \ldots, N, \\
\dot{y}_{j}(t)=y_{j}(t)\left[-r_{j}(t)-e_{j j}(t) y_{j}(t)+\sum_{k=1}^{N} d_{j k}(t) \int_{-\infty}^{t} P_{j k}(t-\sigma) x_{k}(\sigma) \mathrm{d} \sigma\right. & \left.-\sum_{l=1, l \neq j}^{M} e_{j l}(t) \int_{-\infty}^{t} Q_{j l}(t-\sigma) y_{l}(\sigma) \mathrm{d} \sigma\right], \quad j=1,2, \ldots, M .
\end{aligned}
$$

Some sufficient conditions are obtained for the existence of a unique almost-periodic solution of this model. Several examples show that the obtained criteria are new, general and easily verifiable.

Keywords: predator-prey system; Lyapunov functional; almost-periodic solution

2000 Mathematics subject classification: Primary 34D10; 34C25; 34D23; 34K14; 34K20

\section{Introduction}

The Lotka-Volterra system is a rudimentary model in mathematical ecology. The asymptotic behaviour of the Lotka-Volterra competition system with almost-periodic (periodic) coefficients has been studied extensively in $[\mathbf{1}, \mathbf{2}, \mathbf{5}, \mathbf{6}, \mathbf{8}-\mathbf{1 1}, \mathbf{1 4}, \mathbf{1 5}, \mathbf{1 8 - 2 0 , 2 4 , 2 8}]$. Some sufficient conditions are obtained for the uniform persistence, existence and uniqueness of the asymptotic stable almost-periodic (periodic) solution for the Lotka-Volterra competition system. 
The two-species predator-prey Lotka-Volterra system has been investigated extensively in $[\mathbf{3}, \mathbf{1 6}, \mathbf{1 7}]$, and the references cited therein. Some results were obtained for checking existence of the periodic solution and the asymptotic behaviour of these systems. However, few papers have considered the multi-species model. Yang and Xu [25] studied the following periodic system with $M$ predators and $N$ prey:

$$
\left.\begin{array}{l}
\dot{x}_{i}(t)=x_{i}(t)\left[b_{i}(t)-\sum_{k=1}^{N} a_{i k}(t) x_{k}(t)-\sum_{l=1}^{M} c_{i l}(t) y_{l}(t)\right], \quad i=1,2, \ldots, N . \\
\dot{y}_{j}(t)=y_{j}(t)\left[-r_{j}(t)+\sum_{k=1}^{N} d_{j k}(t) x_{k}(t)-\sum_{l=1}^{M} e_{j l}(t) y_{l}(t)\right], \quad j=1,2, \ldots, M .
\end{array}\right\}
$$

where $x_{i}(t)$ denotes the density of prey species $X_{i}$ at time $t$, and $y_{j}(t)$ denotes the density of predator species $Y_{j}$ at time $t$. The coefficients $b_{i}, r_{j}, a_{i k}, c_{i l}, d_{j k}$ and $e_{j l}$ $(i, k=1, \ldots, N ; j, l=1, \ldots, M)$ are non-negative continuous periodic functions defined on $\mathbb{R}$. If (1.1) is autonomous, that is, if these coefficient functions are constants, then $b_{i}$ is the intrinsic growth rate of prey species $X_{i}, r_{j}$ is the death rate of the predator species $Y_{j}$, $a_{i k}$ measures the amount of competition between the prey species $X_{i}$ and $X_{k}(k \neq i$, $i, k=1, \ldots, N), e_{j l}$ measures the amount of competition between the predator species $Y_{j}$ and $Y_{k}(k \neq j, j, k=1, \ldots, M)$, and the constant $\tilde{k}_{i j} \triangleq d_{i j} / c_{i j}$ measures how many of the prey species $X_{i}$ convert into predator species $Y_{j}(i=1, \ldots, N ; j=1, \ldots, M)$. Sufficient conditions for existence and global attractivity of a unique positive periodic solution of system (1.1) were obtained in [25].

Recently, Zhao and Chen [27] have investigated system (1.1) again, allowing the intrinsic growth rate of the prey species to be negative while the total intrinsic growth rate in a period is positive. By using differential inequalities and constructing a Lyapunov function, some sufficient conditions were obtained for existence and global attractivity of a unique positive periodic solution of (1.1). Recently, Xia et al. [23] studied (1.1) with almost-periodic coefficients. Sufficient conditions were obtained for existence and global attractivity of a unique positive almost-periodic solution of (1.1).

The system (1.1) with delay has not been studied so often. Wen [21] considered system (1.1) with several delays, that is

$$
\left.\begin{array}{r}
\dot{x}_{i}(t)=x_{i}(t)\left[b_{i}(t)-a_{i}(t) x_{i}(t)-\sum_{k=1}^{N} a_{i k}(t) x_{k}\left(t-\tau_{i k}\right)-\sum_{l=1}^{M} c_{i l}(t) y_{l}\left(t-\sigma_{i l}\right)\right], \\
i=1,2, \ldots, N, \\
\dot{y}_{j}(t)=y_{j}(t)\left[-r_{j}(t)-e_{j}(t) y_{j}(t)+\sum_{k=1}^{N} d_{j k}(t) x_{k}\left(t-\xi_{j k}\right)-\sum_{l=1}^{M} e_{j l}(t) y_{l}\left(t-\eta_{j l}\right)\right], \\
j=1,2, \ldots, M .
\end{array}\right\}
$$

By means of the comparison theorem and the Lyapunov functional, some sufficient conditions were obtained for existence of the global attractivity of a unique positive periodic solution of system (1.2). 
Recently, Xia and Cao [22] have considered the almost-periodic Lotka-Volterra model with $M$ predators and $N$ prey by 'pure-delay type', that is

$$
\left.\begin{array}{r}
\dot{x}_{i}(t)=x_{i}(t)\left[b_{i}(t)-\sum_{k=1}^{N} a_{i k}(t) x_{k}\left(t-\tau_{i k}(t)\right)-\sum_{l=1}^{M} c_{i l}(t) y_{l}\left(t-\sigma_{i l}(t)\right)\right], \\
i=1,2, \ldots, N, \\
\dot{y}_{j}(t)=y_{j}(t)\left[-r_{j}(t)+\sum_{k=1}^{N} d_{j k}(t) x_{k}\left(t-\xi_{j k}(t)\right)-\sum_{l=1}^{M} e_{j l}(t) y_{l}\left(t-\eta_{j l}(t)\right)\right], \\
j=1,2, \ldots, M .
\end{array}\right\}
$$

By using the concept of eventually uniform $M$-matrix and constructing a suitable Lyapunov functional, a set of sufficient conditions for the existence and global attractivity of a unique positive almost-periodic (periodic) solution of system (1.3) were obtained.

To the best of the authors' knowledge, though Burton and Hutson [4] consider a similar system, there is no paper considering the almost-periodic solutions of the multiplespecies predator-prey model with infinite delays. Therefore, in this paper, we consider the almost-periodic Lotka-Volterra model with $M$ predators and $N$ prey of the integrodifferential equations

$$
\left.\begin{array}{rl}
\dot{x}_{i}(t)=x_{i}(t)\left[b_{i}(t)-a_{i i}(t) x_{i}(t)-\sum_{k=1, k \neq i}^{N} a_{i k}(t) \int_{-\infty}^{t} H_{i k}(t-\sigma) x_{k}(\sigma) \mathrm{d} \sigma\right. \\
\left.-\sum_{l=1}^{M} c_{i l}(t) \int_{-\infty}^{t} K_{i l}(t-\sigma) y_{l}(\sigma) \mathrm{d} \sigma\right], \quad i=1,2, \ldots, N, \\
\dot{y}_{j}(t)=y_{j}(t)\left[-r_{j}(t)-e_{j j}(t) y_{j}(t)+\sum_{k=1}^{N} d_{j k}(t) \int_{-\infty}^{t} P_{j k}(t-\sigma) x_{k}(\sigma) \mathrm{d} \sigma\right. \\
\left.-\sum_{l=1, \neq j}^{M} e_{j l}(t) \int_{-\infty}^{t} Q_{j l}(t-\sigma) y_{l}(\sigma) \mathrm{d} \sigma\right], \quad j=1,2, \ldots, M .
\end{array}\right\}
$$

By using Lebesgue's dominated convergence theorem and constructing a suitable Lyapunov functional, some sufficient conditions are obtained for the existence of a unique almost-periodic solution of system $(E)$.

This paper is organized as follows. In $\S 2$ we use Lebesgue's dominated convergence theorem to show that there exists a bounded solution of system $(E)$ on $\mathbb{R}$. In $\S 3$, by constructing a suitable Lyapunov functional, we shall prove that the solution of system $(E)$ is relatively totally stable. Then some sufficient conditions are obtained for the existence of a unique almost-periodic solution of system $(E)$. Finally, some suitable examples are given to illustrate the main results of this paper.

Throughout this paper, we shall use the following notation.

We always use $i, k=1, \ldots, N ; j, l=1, \ldots, M$, unless otherwise stated. 
If $f$ is an almost-periodic function defined on $\mathbb{R}$, we set

$$
f^{\iota}=\inf _{t \in \mathbb{R}} f(t), \quad f^{\mu}=\sup _{t \in \mathbb{R}} f(t) .
$$

Denote

$$
\begin{aligned}
p_{i} & =\frac{b_{i}^{\mu}}{a_{i i}^{\iota}} \\
q_{j} & =\frac{1}{e_{j j}^{\iota}}\left(\sum_{k=1}^{N} d_{j k}^{\mu} p_{k}-r_{j}^{\iota}\right), \\
\alpha_{i} & =\frac{1}{a_{i i}^{\mu}}\left(b_{i}^{\iota}-\sum_{k=1, k \neq i}^{N} a_{i k}^{\mu} p_{k}-\sum_{l=1}^{M} c_{i l}^{\mu} q_{l}\right), \\
\beta_{j} & =\frac{1}{e_{j j}^{\mu}}\left(-r_{j}^{\mu}+\sum_{k=1}^{N} d_{j k}^{\iota} \alpha_{k}-\sum_{l=1, l \neq j}^{M} c_{j l}^{\mu} q_{l}\right), \\
x^{*} & =\max _{i, j}\left\{p_{i}, q_{j}\right\}, \quad x_{*}=\min _{i, j}\left\{\alpha_{i}, \beta_{j}\right\} .
\end{aligned}
$$

It is obvious that if $q_{j}>0$, then $\alpha_{i}<p_{i}$, and if $q_{j}>0, \alpha_{i}>0$, then $\beta_{j}<q_{j}$.

We denote by $\mathbb{R}^{N+M}$ the $(N+M)$-dimensional real Euclidean space and by $|x|$ the norm of $x \in \mathbb{R}^{N+M}$.

Let

$$
\begin{aligned}
& \boldsymbol{B}=\{\Phi=(\phi, \psi) \mid \Phi:(-\infty, 0] \mapsto \mathbb{R}^{N+M}, \\
&\Phi(t) \text { is a bounded and uniformly continuous function }\}
\end{aligned}
$$

(see $[\mathbf{1 2}, \mathbf{1 3}]$ ). For $\Phi \in \boldsymbol{B}$, we set $\|\Phi\|=\sup _{s \leqslant 0}|\Phi(s)|$.

For any $\Phi, \Psi \in \boldsymbol{B}$, we set

$$
\begin{aligned}
\rho_{m}(\Phi, \Psi) & =\sup _{-m \leqslant s \leqslant 0}|\Phi(s)-\Psi(s)|, \\
\rho(\Phi, \Psi) & =\sum_{m=1}^{\infty} \frac{\rho_{m}(\Phi, \Psi)}{2^{m}\left(1+\rho_{m}(\Phi, \Psi)\right)} .
\end{aligned}
$$

Obviously, $\rho\left(\Phi_{n}, \Phi\right) \mapsto 0$, as $n \rightarrow \infty$, if and only if $\Phi_{n}(s) \rightarrow \Phi(s)$ as $n \rightarrow \infty$ uniformly on each bounded subset of $(-\infty, 0]$.

For any function $x: R \mapsto \mathbb{R}^{N+M}$ and $t \in \mathbb{R}$, we define a function $x^{t}:(-\infty, 0] \mapsto \mathbb{R}^{N+M}$ by $x^{t}(s)=x(t+s)$ for $s \leqslant 0$.

Throughout this paper, we suppose that the following conditions are satisfied.

$\left(\mathrm{H}_{1}\right) b_{i}, r_{j}, a_{i k}, c_{i l}, d_{j k}$ and $e_{j l}$ are non-negative almost-periodic functions defined on $\mathbb{R}$ with $\inf _{t \in \mathbb{R}} a_{i i}(t)>0, \inf _{t \in \mathbb{R}} e_{j j}(t)>0$. 
$\left(\mathrm{H}_{2}\right) H_{i k}, K_{i l}, P_{j k}$ and $Q_{j l}(t)$ are non-negative functions, and

$$
\begin{aligned}
& \int_{0}^{\infty} H_{i k}(s) \mathrm{d} s=1, \quad \int_{0}^{\infty} s H_{i k}(s) \mathrm{d} s<\infty ; \\
& \int_{0}^{\infty} K_{i l}(s) \mathrm{d} s=1, \quad \int_{0}^{\infty} s K_{i l}(s) \mathrm{d} s<\infty ; \\
& \int_{0}^{\infty} P_{j k}(s) \mathrm{d} s=1, \quad \int_{0}^{\infty} s P_{j k}(s) \mathrm{d} s<\infty ; \\
& \int_{0}^{\infty} Q_{j l}(s) \mathrm{d} s=1, \quad \int_{0}^{\infty} s Q_{j l}(s) \mathrm{d} s<\infty .
\end{aligned}
$$

\section{Existence of bounded solutions}

In the following we will state some lemmas that will be used in the proof of Theorem 2.4. Since we are interested in the positive solutions of the system, we assume that system $(E)$ is supplemented with initial conditions of the form

$$
\left.\begin{array}{lll}
x_{i}(s)=\phi_{i}(s) \geqslant 0, \quad s \leqslant 0 ; & \sup _{s \leqslant 0} \phi_{i}(s)<\infty ; & \phi_{i}(0)>0 . \\
y_{j}(s)=\psi_{j}(s) \geqslant 0, \quad s \leqslant 0 ; & \sup _{s \leqslant 0} \psi_{j}(s)<\infty ; & \psi_{j}(0)>0 .
\end{array}\right\}
$$

Lemma 2.1. Both the positive and non-negative cones of $\mathbb{R}^{N+M}$ are invariant with respect to $(E)$.

Proof. Since

$$
\begin{array}{r}
x_{i}(t)=x_{i}\left(t_{0}\right) \exp \left\{\int _ { t _ { 0 } } ^ { t } \left[b_{i}(s)-a_{i i}(s) x_{i}(s)-\sum_{k=1, k \neq i}^{N} a_{i k}(s) \int_{-\infty}^{s} H_{i k}(s-\sigma) x_{k}(\sigma) \mathrm{d} \sigma\right.\right. \\
\left.\left.-\sum_{l=1}^{M} c_{i l}(s) \int_{-\infty}^{s} K_{i l}(s-\sigma) y_{l}(\sigma) \mathrm{d} \sigma\right] \mathrm{d} s\right\}, \\
\begin{aligned}
y_{j}(t)=y_{j}\left(t_{0}\right) \exp \left\{\int _ { t _ { 0 } } ^ { t } \left[-r_{j}(s)-e_{j j}(s) y_{j}(s)\right.\right. & +\sum_{k=1}^{N} d_{j k}(s) \int_{-\infty}^{s} P_{j k}(s-\sigma) x_{k}(\sigma) \mathrm{d} \sigma \\
& \left.\left.-\sum_{l=1, \neq j}^{M} e_{j l}(s) \int_{-\infty}^{s} Q_{j l}(s-\sigma) y_{l}(\sigma) \mathrm{d} \sigma\right] \mathrm{d} s\right\}
\end{aligned}
\end{array}
$$

the assertion of Lemma 2.1 follows immediately, for all $t \in\left[t_{0},+\infty\right)$.

Lemma 2.2. If $a>0, b>0$ and $\mathrm{d} x(t) / \mathrm{d} t \leqslant x(t)[b-a x(t)]$, for $t \geqslant t_{0}$, then

$$
x(t) \leqslant \frac{b}{a}\left[1+\left(\frac{b}{a x\left(t_{0}\right)}-1\right) \mathrm{e}^{-b t}\right]^{-1}, \quad t \geqslant t_{0} .
$$

Moreover, if $0<x\left(t_{0}\right) \leqslant b / a$, then $0<x(t) \leqslant b / a$. 
If $a>0, b>0$ and $\mathrm{d} x(t) / \mathrm{d} t \geqslant x(t)[b-a x(t)]$, for $t \geqslant t_{0}$, then

$$
x(t) \geqslant \frac{b}{a}\left[1+\left(\frac{b}{a x\left(t_{0}\right)}-1\right) \mathrm{e}^{-b t}\right]^{-1}, \quad t \geqslant t_{0} .
$$

Moreover, if $x\left(t_{0}\right) \geqslant b / a>0$, then $x(t) \geqslant b / a>0$.

Proof. We give a proof for the first case. From $\mathrm{d} x(t) / \mathrm{d} t \leqslant x(t)[b-a x(t)]$, we have

$$
\frac{\mathrm{d} x^{-1}(t)}{\mathrm{d} t} \geqslant a-b x^{-1}(t), \quad \frac{\mathrm{d}\left(\mathrm{e}^{b t} x^{-1}(t)\right)}{\mathrm{d} t} \geqslant a \mathrm{e}^{b t} .
$$

Hence, we have

$$
\mathrm{e}^{b t} x^{-1}(t)-x^{-1}\left(t_{0}\right) \geqslant \frac{a}{b}\left(\mathrm{e}^{b t}-1\right)
$$

Moreover, we have

$$
x^{-1}(t) \geqslant \mathrm{e}^{-b t} x^{-1}\left(t_{0}\right)+\frac{b}{a}\left(1-\mathrm{e}^{-b t}\right)=\frac{a}{b}\left[1+\left(\frac{b}{a x\left(t_{0}\right)}-1\right) \mathrm{e}^{-b t}\right] .
$$

Therefore, we obtain

$$
x(t) \leqslant \frac{b}{a}\left[1+\left(\frac{b}{a x\left(t_{0}\right)}-1\right) \mathrm{e}^{-b t}\right]^{-1} .
$$

Then it is not difficult to derive that if $0<x\left(t_{0}\right) \leqslant b / a$, then $0<x(t) \leqslant b / a$. This completes the proof of Lemma 2.2.

Lemma 2.3. Let $\Phi=(\phi, \psi) \in \boldsymbol{B}$ satisfy $\alpha_{i} \leqslant \phi_{i}(s) \leqslant p_{i}, \beta_{j} \leqslant \psi_{j}(s) \leqslant q_{j}$ for all $s \leqslant 0$, and $F=(x, y)$ be the solution of $(E)$ through $\left(t_{0}, \Phi\right)$. If $(E)$ satisfies $\alpha_{i}>0, \beta_{j}>0$ and $q_{j}>0$, then

$$
\alpha_{i} \leqslant x_{i}(t) \leqslant p_{i}, \quad \beta_{j} \leqslant y_{j}(t) \leqslant q_{j}, \quad \text { for all } t \geqslant t_{0} .
$$

Proof. From the first equation of system $(E)$, we have $\dot{x}_{i} \leqslant x_{i}\left(b_{i}^{\mu}-a_{i i}^{\iota} x_{i}\right)$, so if

$$
0<x_{i}\left(t_{0}\right) \leqslant p_{i}
$$

then from Lemma 2.2 we have

$$
x_{i}(t) \leqslant p_{i} .
$$

From (2.2) and the second equation of system $(E)$, we obtain

$$
\dot{y}_{j} \leqslant y_{j}\left[-r_{j}^{\iota}+\sum_{k=1}^{N} d_{j k}^{\mu} p_{k} \int_{-\infty}^{t} P_{j k}(t-\sigma) \mathrm{d} \sigma-e_{j j}^{\iota} y_{j}\right] .
$$

Since

$$
\int_{-\infty}^{t} P_{j k}(t-\sigma) \mathrm{d} \sigma=\int_{0}^{\infty} P_{j k}(s) \mathrm{d} s=1
$$

we obtain

$$
\dot{y}_{j} \leqslant y_{j}\left[\left(-r_{j}^{\iota}+\sum_{k=1}^{N} d_{j k}^{\mu} p_{k}\right)-e_{j j}^{\iota} y_{j}\right] .
$$


Hence, if

$$
0<y_{j}\left(t_{0}\right) \leqslant q_{j}
$$

then from Lemma 2.2 we have

$$
y_{j}(t) \leqslant q_{j} .
$$

Now the first equation of $(E),(2.2),(2.3)$ and $\left(\mathrm{H}_{2}\right)$ lead to

$$
\begin{aligned}
\dot{x}_{i} & \geqslant x_{i}\left[b_{i}^{\iota}-\sum_{k=1, k \neq i}^{N} a_{i k}^{\mu} p_{k} \int_{-\infty}^{t} H_{i k}(t-\sigma) \mathrm{d} \sigma-\sum_{l=1}^{M} c_{i l}^{\mu} q_{l} \int_{-\infty}^{t} K_{i l}(t-\sigma) \mathrm{d} \sigma-a_{i i}^{\mu} x_{i}\right] \\
& =x_{i}\left[b_{i}^{\iota}-\sum_{k=1, k \neq i}^{N} a_{i k}^{\mu} p_{k} \int_{0}^{\infty} H_{i k}(s) \mathrm{d} s-\sum_{l=1}^{M} c_{i l}^{\mu} q_{l} \int_{0}^{\infty} K_{i l}(s) \mathrm{d} s-a_{i i}^{\mu} x_{i}\right] \\
& =x_{i}\left[\left(b_{i}^{\iota}-\sum_{k=1, k \neq i}^{N} a_{i k}^{\mu} p_{k}-\sum_{l=1}^{M} c_{i l}^{\mu} q_{l}\right)-a_{i i}^{\mu} x_{i}\right],
\end{aligned}
$$

which implies that if $x_{i}\left(t_{0}\right) \geqslant \alpha_{i}$ holds, from Lemma 2.2 we have

$$
x_{i}(t) \geqslant \alpha_{i} .
$$

If $\alpha_{i}>0$, then from $(E),(2.2),(2.3)$ and $\left(\mathrm{H}_{2}\right)$, by a similar discussion to that above, we obtain

$$
\dot{y}_{j} \geqslant y_{j}\left[\left(-r_{j}^{\mu}+\sum_{k=1}^{N} d_{j k}^{\iota} \alpha_{k}-\sum_{l=1, l \neq j}^{M} e_{j l}^{\mu} q_{l}\right)-e_{j j}^{\mu} y_{j}\right],
$$

which implies that if $y_{j}\left(t_{0}\right) \geqslant \beta_{j}$ holds, from Lemma 2.2 we obtain

$$
y_{j}(t) \geqslant \beta_{j} .
$$

If $\alpha_{i}>0, \beta_{j}>0$ and $q_{j}>0$, it is obvious that $0<\alpha_{i}<p_{i}, 0<\beta_{j}<q_{j}$. Therefore, we have $\alpha_{i} \leqslant x_{i}(t) \leqslant p_{i}, \beta_{j} \leqslant y_{j}(t) \leqslant q_{j}$, for all $t \geqslant t_{0}$. This completes the proof of Lemma 2.3 .

We denote by $S(E)$ the set of all solutions $F=(x, y)$ of $(E)$ on $\mathbb{R}$ satisfying $\alpha_{i} \leqslant$ $x_{i}(t) \leqslant p_{i}, \beta_{j} \leqslant y_{j}(t) \leqslant q_{j}$ for all $t \in \mathbb{R}$.

Theorem 2.4. If (E) satisfies

$\left(\mathrm{H}_{3}\right) \alpha_{i}>0, \beta_{j}>0, q_{j}>0$,

then $S(E) \neq \emptyset$.

Proof. By $\left(\mathrm{H}_{1}\right)$, there exists a sequence $\left\{t_{n}\right\}, t_{n} \rightarrow \infty$ as $n \rightarrow \infty$, such that

$$
\left.\begin{array}{rl}
b_{i}\left(t+t_{n}\right) & \rightarrow b_{i}(t), \\
r_{j}\left(t+t_{n}\right) & \rightarrow r_{j}(t), \\
a_{i k}\left(t+t_{n}\right) & \rightarrow a_{i k}(t), \\
c_{i l}\left(t+t_{n}\right) & \rightarrow c_{i l}(t), \\
d_{j k}\left(t+t_{n}\right) & \rightarrow d_{j k}(t), \\
e_{j l}\left(t+t_{n}\right) & \rightarrow e_{j l}(t),
\end{array}\right\} \quad \text { as } n \rightarrow \infty \text { uniformly on } \mathbb{R} .
$$


Let $F=(x, y)$ be a solution of $(E)$ through $\left(t_{0}, \Phi\right) \in \mathbb{R} \times \boldsymbol{B}$ satisfying $\alpha_{i} \leqslant x_{i}(t) \leqslant p_{i}$, $\beta_{j} \leqslant y_{j}(t) \leqslant q_{j}$ for all $t \geqslant t_{0}$, whose existence was ensured by Lemma 2.3. Obviously, the sequence $\left\{F\left(t+t_{n}\right)\right\}$ is uniformly bounded and equicontinuous on each bounded subset of $\mathbb{R}$. Therefore, by Ascoli's theorem and a diagonalization procedure, we may assume that the sequence $\left\{F\left(t+t_{n}\right)\right\}$ converges to a continuous function $U(t)=(u(t), v(t))=$ $\left(u_{1}(t), \ldots, u_{N}(t), v_{1}(t), \ldots, v_{M}(t)\right)$ as $n \rightarrow \infty$ uniformly on each bounded subset of $\mathbb{R}$. Let a $\tau \in \mathbb{R}$ be given. We may assume that $t_{n}+\tau \geqslant t_{0}$ for all $n$. For $t \geqslant t_{0}$, we have

$$
\begin{aligned}
& x_{i}\left(t+t_{n}+\tau\right)-x_{i}\left(t_{n}+\tau\right) \\
& =\int_{t_{n}+\tau}^{t+t_{n}+\tau} x_{i}(s)\left\{b_{i}(s)-a_{i i}(s) x_{i}(s)-\sum_{k=1, k \neq i}^{N} a_{i k}(s) \int_{-\infty}^{s} H_{i k}(s-\sigma) x_{k}(\sigma) \mathrm{d} \sigma\right. \\
& \left.-\sum_{l=1}^{M} c_{i l}(s) \int_{-\infty}^{s} K_{i l}(s-\sigma) y_{l}(\sigma) \mathrm{d} \sigma\right\} \mathrm{d} s \\
& =\int_{\tau}^{t+\tau} x_{i}\left(\bar{s}+t_{n}\right)\left\{b_{i}\left(\bar{s}+t_{n}\right)-a_{i i}\left(\bar{s}+t_{n}\right) x_{i}\left(\bar{s}+t_{n}\right)\right. \\
& -\sum_{l=1, l \neq i}^{N} a_{i l}\left(\bar{s}+t_{n}\right) \int_{-\infty}^{\bar{s}+t_{n}} H_{i k}\left(\bar{s}+t_{n}-\sigma\right) x_{k}(\sigma) \mathrm{d} \sigma \\
& \left.-\sum_{k=1}^{M} c_{i k}(s) \int_{-\infty}^{\bar{s}+t_{n}} K_{i k}\left(\bar{s}+t_{n}-\sigma\right) y_{k}(\sigma) \mathrm{d} \sigma\right\} \mathrm{d} \bar{s} \\
& y_{j}\left(t+t_{n}+\tau\right)-y_{j}\left(t_{n}+\tau\right) \\
& =\int_{t_{n}+\tau}^{t+t_{n}+\tau} y_{j}(s)\left\{-r_{j}(s)-e_{j j}(s) y_{j}(s)+\sum_{k=1}^{N} d_{j k}(s) \int_{-\infty}^{s} P_{j k}(s-\sigma) x_{k}(\sigma) \mathrm{d} \sigma\right. \\
& \left.-\sum_{l=1, l \neq i}^{M} e_{j l}(s) \int_{-\infty}^{s} Q_{j l}(s-\sigma) y_{l}(\sigma) \mathrm{d} \sigma\right\} \mathrm{d} s \\
& =\int_{\tau}^{t+\tau} x_{i}\left(\bar{s}+t_{n}\right)\left\{-r_{j}\left(\bar{s}+t_{n}\right)-e_{j j}\left(\bar{s}+t_{n}\right) y_{j}\left(\bar{s}+t_{n}\right)\right. \\
& +\sum_{k=1}^{N} d_{j k}\left(\bar{s}+t_{n}\right) \int_{-\infty}^{\bar{s}+t_{n}} P_{j k}\left(\bar{s}+t_{n}-\sigma\right) x_{k}(\sigma) \mathrm{d} \sigma \\
& \left.-\sum_{l=1, l \neq j}^{M} e_{j l}(s) \int_{-\infty}^{\bar{s}+t_{n}} Q_{j l}\left(\bar{s}+t_{n}-\sigma\right) y_{l}(\sigma) \mathrm{d} \sigma\right\} \mathrm{d} \bar{s} .
\end{aligned}
$$

We note that

$$
\begin{aligned}
\int_{-\infty}^{\bar{s}+t_{n}} H_{i k}\left(\bar{s}+t_{n}-\sigma\right) x_{k}(\sigma) \mathrm{d} \sigma & =\int_{0}^{\infty} H_{i k}(\bar{\sigma}) x_{k}\left(\bar{s}+t_{n}-\bar{\sigma}\right) \mathrm{d} \bar{\sigma}, \\
H_{i k}(\bar{\sigma}) x_{k}\left(\bar{s}+t_{n}-\bar{\sigma}\right) & \rightarrow H_{i k}(\bar{\sigma}) u_{k}(\bar{s}-\bar{\sigma}) \quad \text { as } n \rightarrow \infty
\end{aligned}
$$


and that

$$
\left|H_{i k}(\bar{\sigma}) x_{k}\left(\bar{s}+t_{n}-\bar{\sigma}\right)\right| \leqslant H_{i k}(\bar{\sigma}) p_{k} \quad \text { for } \bar{\sigma} \geqslant t_{0}, \bar{s} \in[\tau, t+\tau] .
$$

By $\left(\mathrm{H}_{2}\right)$ and Lebesgue's dominated convergence theorem, it follows that

$\lim _{n \rightarrow \infty} \int_{0}^{\infty} H_{i k}(\bar{\sigma}) x_{k}\left(\bar{s}+t_{n}-\bar{\sigma}\right) \mathrm{d} \bar{\sigma}=\int_{0}^{\infty} H_{i k}(\bar{\sigma}) u_{k}(\bar{s}-\bar{\sigma}) \mathrm{d} \bar{\sigma}=\int_{-\infty}^{\bar{s}} H_{i k}(\bar{s}-\sigma) u_{k}(\sigma) \mathrm{d} \sigma$.

Similarly, by $\left(\mathrm{H}_{2}\right)$ and Lebesgue's dominated convergence theorem, we obtain

$$
\begin{aligned}
\lim _{n \rightarrow \infty} \int_{-\infty}^{\bar{s}+t_{n}} K_{i l}\left(\bar{s}+t_{n}-\sigma\right) y_{l}(\sigma) \mathrm{d} \sigma & =\int_{0}^{\infty} K_{i l}(\bar{\sigma}) v_{l}(\bar{s}-\bar{\sigma}) \mathrm{d} \bar{\sigma} \\
& =\int_{-\infty}^{\bar{s}} K_{i l}(\bar{s}-\sigma) v_{l}(\sigma) \mathrm{d} \sigma \\
\lim _{n \rightarrow \infty} \int_{-\infty}^{\bar{s}+t_{n}} P_{j k}\left(\bar{s}+t_{n}-\sigma\right) x_{k}(\sigma) \mathrm{d} \sigma & =\int_{0}^{\infty} P_{j k}(\bar{\sigma}) u_{k}(\bar{s}-\bar{\sigma}) \mathrm{d} \bar{\sigma} \\
& =\int_{-\infty}^{\bar{s}} P_{j k}(\bar{s}-\sigma) u_{k}(\sigma) \mathrm{d} \sigma \\
\lim _{n \rightarrow \infty} \int_{-\infty}^{\bar{s}+t_{n}} Q_{j l}\left(\bar{s}+t_{n}-\sigma\right) y_{l}(\sigma) \mathrm{d} \sigma & =\int_{0}^{\infty} Q_{j l}(\bar{\sigma}) v_{l}(\bar{s}-\bar{\sigma}) \mathrm{d} \bar{\sigma} \\
& =\int_{-\infty}^{\bar{s}} Q_{j l}(\bar{s}-\sigma) v_{l}(\sigma) \mathrm{d} \sigma .
\end{aligned}
$$

From (2.8)-(2.11), and letting $n \rightarrow \infty$ in (2.6) and (2.7), respectively, we have

$$
\begin{gathered}
u_{i}(t+\tau)-u_{i}(\tau) \\
=\int_{\tau}^{t+\tau} u_{i}(\bar{s})\left\{b_{i}(\bar{s})-a_{i i}(\bar{s}) u_{i}(\bar{s})-\sum_{k=1, k \neq i}^{N} a_{i k}(\bar{s}) \int_{-\infty}^{\bar{s}} H_{i k}(\bar{s}-\sigma) u_{k}(\sigma) \mathrm{d} \sigma\right. \\
\left.-\sum_{l=1}^{M} c_{i l}(\bar{s}) \int_{-\infty}^{\bar{s}} K_{i l}(\bar{s}-\sigma) v_{l}(\sigma) \mathrm{d} \sigma\right\} \mathrm{d} \bar{s}, \\
\begin{aligned}
v_{j}(t+\tau)-v_{j}(\tau) \\
=\int_{\tau}^{t+\tau} v_{j}(\bar{s})\left\{-r_{j}(\bar{s})-e_{j j}(\bar{s}) v_{j}(\bar{s})+\sum_{k=1}^{N} d_{j k}(\bar{s}) \int_{-\infty}^{\bar{s}} P_{j k}(\bar{s}-\sigma) u_{k}(\sigma) \mathrm{d} \sigma\right. \\
\left.-\sum_{l=1, l \neq j}^{M} e_{j l}(\bar{s}) \int_{-\infty}^{\bar{s}} Q_{j l}(\bar{s}-\sigma) v_{l}(\sigma) \mathrm{d} \sigma\right\} \mathrm{d} \bar{s},
\end{aligned}
\end{gathered}
$$

for all $t \geqslant t_{0}$. Since $\tau \in \mathbb{R}$ is arbitrarily given,

$$
U(t)=(u(t), v(t))=\left(u_{1}(t), \ldots, u_{N}(t), v_{1}(t), \ldots, v_{M}(t)\right)
$$


is a solution of system $(E)$ on $\mathbb{R}$. It is clear that $\alpha_{i} \leqslant u_{i}(t) \leqslant p_{i}, \beta_{j} \leqslant v_{j}(t) \leqslant q_{j}$ for all $t \in \mathbb{R}$. Thus, $U(t) \in S(E)$. This completes the proof of the theorem.

By repeating almost the same argument as in the proof of Theorem 2.4, we can also prove the following theorem.

Theorem 2.5. Let $U(t) \in S(E)$, and let a sequence $\left\{t_{n}\right\}, t_{n} \geqslant 0$, be given. Suppose that, for some functions $\bar{b}_{i}, \bar{r}_{j}, \bar{a}_{i k}, \bar{c}_{i l}, \bar{d}_{j k}$ and $\bar{e}_{j l}$,

(i) $b_{i}\left(t+t_{n}\right) \rightarrow \bar{b}_{i}(t), r_{j}\left(t+t_{n}\right) \rightarrow \bar{r}_{j}(t), a_{i k}\left(t+t_{n}\right) \rightarrow \bar{a}_{i k}(t), c_{i l}\left(t+t_{n}\right) \rightarrow \bar{c}_{i l}(t)$, $d_{j k}\left(t+t_{n}\right) \rightarrow \bar{d}_{j k}(t), e_{j l}\left(t+t_{n}\right) \rightarrow \bar{e}_{j l}(t)$, as $n \rightarrow \infty$, uniformly on $t \in \mathbb{R}$;

(ii) $U\left(t+t_{n}\right) \rightarrow \bar{U}(t)$ as $n \rightarrow \infty$ uniformly on a subset of $\mathbb{R}$.

Then $\bar{U}(t) \in S(\bar{E})$, where $S(\bar{E})$ is the set of solutions $W=(w, z)$ of the system

$$
\left.\begin{array}{r}
\dot{w}_{i}(t)=w_{i}(t)\left[\bar{b}_{i}(t)-\bar{a}_{i i}(t) w_{i}(t)-\sum_{k=1, k \neq i}^{N} \bar{a}_{i k}(t) \int_{-\infty}^{t} H_{i k}(t-\sigma) w_{k}(\sigma) \mathrm{d} \sigma\right. \\
\left.-\sum_{l=1}^{M} \bar{c}_{i l}(t) \int_{-\infty}^{t} K_{i l}(t-\sigma) z_{l}(\sigma) \mathrm{d} \sigma\right], \\
\dot{z}_{j}(t)=z_{j}(t)\left[-\bar{r}_{j}(t)-\bar{e}_{j j}(t) z_{j}(t)+\sum_{k=1}^{N} \bar{d}_{j k}(t)\right. \\
\int_{-\infty}^{t} P_{j k}(t-\sigma) w_{k}(\sigma) \mathrm{d} \sigma \\
\left.-\sum_{l=1, l \neq j}^{M} \bar{e}_{j l}(t) \int_{-\infty}^{t} Q_{j l}(t-\sigma) z_{l}(\sigma) \mathrm{d} \sigma\right]
\end{array}\right\}
$$

on $\mathbb{R}$ satisfying $\alpha_{i} \leqslant w_{i}(t) \leqslant p_{i}, \beta_{j} \leqslant z_{j}(t) \leqslant q_{j}$ for all $t \in \mathbb{R}$.

When (i) and (ii) hold, we write $(\bar{U}, \bar{E}) \in \Omega(U, E)$. Based on Theorems 2.4 and 2.5, we will discuss the existence of the almost-periodic solution of system $(E)$ in $\S 3$.

\section{Existence of a unique almost-periodic solution}

We now state some definitions and lemmas which will be used in the proof of our main theorem (Theorem 3.8). The method is as follows: by using a series of definitions (especially the concept of asymptotic almost-periodicity), we prove first that system $(E)$ has an asymptotic almost-periodic solution and then that system $(E)$ has a unique almostperiodic solution.

Definition 3.1. A function $U \in S(E)$ is said to be relatively uniformly stable (RUS) in $\Omega(E)$ if, for any $\varepsilon>0$, there exists a $\delta(\varepsilon)>0$ with the property that, for any $t_{0} \geqslant 0$, any $(\bar{U}, \bar{E}) \in \Omega(U, E)$ and any $\bar{W} \in S(\bar{E})$ satisfying $\rho\left(\bar{U}^{t_{0}}, \bar{W}^{t_{0}}\right)<\delta(\varepsilon)$, we have $\rho\left(\bar{U}^{t}, \bar{W}^{t}\right)<\varepsilon$ for all $t \geqslant t_{0}$.

Definition 3.2. A function $U \in S(E)$ is said to be relatively weakly uniformly asymptotically stable (RWUAS) in $\Omega(E)$ if $U(t)$ is RUS in $\Omega(E)$, and if $\rho\left(\bar{U}^{t}, \bar{W}^{t}\right) \rightarrow 0$ as $t \rightarrow \infty$ for all $(\bar{U}, \bar{E}) \in \Omega(U, E)$ and all $\bar{W} \in S(\bar{E})$. 
Definition 3.3. A function $U \in S(E)$ is said to be relatively totally stable (RTS) for $(E)$ if, for any $\varepsilon>0$, there exists a $\delta(\varepsilon)>0$ with the property that if $t_{0} \geqslant 0$, $\rho\left(F^{t_{0}}, U^{t_{0}}\right)<\delta(\varepsilon)$ and $G=(f, g): R \mapsto \mathbb{R}^{N+M}$ is any continuous function satisfying $\sup _{t \in \mathbb{R}}|G(t)|<\delta(\varepsilon)$, then we have $\rho\left(F^{t}, U^{t}\right)<\delta(\varepsilon)$ for all $t \geqslant t_{0}$, where $F=(x, y)$ is any solution of the system

$$
\left.\begin{array}{r}
\dot{x}_{i}(t)=x_{i}(t)\left[b_{i}(t)-a_{i i}(t) x_{i}(t)-\sum_{k=1, k \neq i}^{N} a_{i k}(t) \int_{-\infty}^{t} H_{i k}(t-\sigma) x_{k}(\sigma) \mathrm{d} \sigma\right. \\
\left.-\sum_{l=1}^{M} c_{i l}(t) \int_{-\infty}^{t} K_{i l}(t-\sigma) y_{l}(\sigma) \mathrm{d} \sigma\right]+f_{i}(t), \\
\dot{y}_{j}(t)=y_{j}(t)\left[-r_{j}(t)-e_{j j}(t) y_{j}(t)+\sum_{k=1}^{N} d_{j k}(t) \int_{-\infty}^{t} P_{j k}(t-\sigma) x_{k}(\sigma) \mathrm{d} \sigma\right. \\
\left.-\sum_{l=1, j}^{M} e_{j l}(t) \int_{-\infty}^{t} Q_{j l}(t-\sigma) y_{l}(\sigma) \mathrm{d} \sigma\right]+g_{i}(t)
\end{array}\right\}
$$

on $\mathbb{R}$ satisfying $\alpha_{i} \leqslant x_{i}(t) \leqslant p_{i}, \beta_{j} \leqslant y_{j}(t) \leqslant q_{j}$ for all $t \in \mathbb{R}$.

Definition 3.4 (Fink [7]). A continuous function is asymptotic almost periodic if and only if there is an almost-periodic function $p$ and a continuous function $q$ defined on $\mathbb{R}^{+}$with $\lim _{t \rightarrow \infty} q(t)=0$ such that

$$
f(t)=p(t)+q(t)
$$

Definition 3.5 (Fink [7]). For every sequence $\left\{h_{k}\right\}$, where $h_{k}>0$ and $h_{k} \rightarrow \infty$ as $k \rightarrow \infty$, if there exists a subsequence $\left\{h_{k j}\right\}$ such that $f\left(t+h_{k j}\right)$ is uniformly convergent on $[0,+\infty)$, then it is said that $f$ has property $\boldsymbol{L}$.

Lemma 3.6. If $U \in S(E)$ is $R W U A S$ in $\Omega(E)$, then it is RTS for $(E)$.

Proof. We give the proof for completeness, although it is similar to the one for $[\mathbf{1 8}$, Lemma 4]. Suppose the contrary. There then exists an $\varepsilon>0$, sequences $\left\{\varepsilon_{n}\right\}, 0<$ $\varepsilon_{n}<\varepsilon$, and $\varepsilon_{n} \rightarrow 0$ as $n \rightarrow \infty,\left\{s_{n}\right\},\left\{t_{n}\right\}, t_{n} \geqslant s_{n} \geqslant 0,\left\{G_{n}\right\}$ and $\left\{F^{n}\right\}$ such that $G_{n}: R \rightarrow R^{N+M}$ is a continuous function satisfying $\sup _{t \in \mathbb{R}}\left|G_{n}(t)\right|<\varepsilon$ and that

$$
\rho\left(U^{s_{n}},\left(F^{n}\right)^{s_{n}}\right)<\varepsilon_{n}, \quad \rho\left(U^{t_{n}},\left(F^{n}\right)^{t_{n}}\right)=\varepsilon, \quad \rho\left(U^{t},\left(F^{n}\right)^{t}\right)<\varepsilon, \quad t \in\left[s_{n}, t_{n}\right),
$$

where $F^{n}$ is a solution of $\left(E_{G_{n}}\right)$ on $\mathbb{R}$ satisfying $\alpha_{i} \leqslant\left(x^{n}\right)_{i}(t) \leqslant p_{i}, \beta_{j} \leqslant\left(y^{n}\right)_{j}(t) \leqslant q_{j}$ for all $t \in \mathbb{R}$.

Furthermore, by (3.1) we can choose a sequence $\left\{\tau_{n}\right\}, s_{n}<\tau_{n}<t_{n}$, so that

$$
\rho\left(U^{\tau_{n}},\left(F^{n}\right)^{\tau_{n}}\right)=\frac{1}{2} \delta\left(\frac{1}{2} \varepsilon\right)
$$

and

$$
\frac{1}{2} \delta\left(\frac{1}{2} \varepsilon\right) \leqslant \rho\left(U^{t},\left(F^{n}\right)^{t}\right) \leqslant \varepsilon, \quad t \in\left[\tau_{n}, t_{n}\right]
$$


where $\delta(\cdot)$ is the number given in Definition 3.1. We may assume that $U\left(t+\tau_{n}\right) \rightarrow \bar{U}(t)$ as $n \rightarrow \infty$ on each bounded subset of $\mathbb{R}$ for a continuous function $\bar{U}(t)$ and that $(\bar{U}, \bar{E}) \in \Omega(U, E)$. Moreover, we may assume that $F^{n}\left(\tau_{n}+t\right) \rightarrow \bar{W}(t)$ as $n \rightarrow \infty$ uniformly on any bounded subset of $\mathbb{R}$ for a continuous function $\bar{W}$, since the sequence $\left\{F^{n}\left(\tau_{n}+t\right)\right\}$ is uniformly bounded and equicontinuous on $\mathbb{R}$. Then, the same argument as in the proof of Theorem 2.4 shows that $\bar{W} \in S(\bar{E})$. Now, suppose that $t_{n}-\tau_{n} \rightarrow \infty$ as $n \rightarrow \infty$. Letting $n \rightarrow \infty$ in (3.3), we have $\frac{1}{2} \delta\left(\frac{1}{2} \varepsilon\right) \leqslant \rho\left(\bar{U}^{t}, \bar{W}^{t}\right) \leqslant \varepsilon$ for all $t \geqslant 0$. On the other hand, $\rho\left(\bar{U}^{t}, \bar{W}^{t}\right) \rightarrow 0$ as $t \rightarrow \infty$, since $U$ is RWUAS in $\Omega(E)$. This is a contradiction. Thus, taking a subsequence if necessary, we may assume that $t_{n}-\tau_{n} \rightarrow r<\infty$ as $n \rightarrow \infty$. Let $n \rightarrow \infty$ in (3.2). We obtain $\rho\left(\bar{U}^{0}, \bar{W}^{0}\right)=\frac{1}{2} \delta\left(\frac{1}{2} \varepsilon\right)<\delta\left(\frac{1}{2} \varepsilon\right)$, and hence $\rho\left(\bar{U}^{t}, \bar{W}^{t}\right)<\frac{1}{2} \varepsilon$ for all $t \geqslant 0$, because $U$ is RUS in $\Omega(E)$. On the other hand, from (3.1) we have $\rho\left(\bar{U}^{r}, \bar{W}^{r}\right)=\varepsilon$, which is a contradiction. This completes the proof of Lemma 3.6.

Lemma 3.7 (see Chapter 1 in Fink [7] or Theorem 9.3(1) in Burton and Hutson [4]). The asymptotic almost-periodicity of $f(t)$ is equivalent to $f(t)$ having property $\boldsymbol{L}$.

We now state our main result on the existence of a unique almost-periodic solution of system $(E)$.

Theorem 3.8. If system $(E)$ satisfies $\left(\mathrm{H}_{1}\right)-\left(\mathrm{H}_{3}\right)$ and

$\left(\mathrm{H}_{4}\right)$ there exist strictly positive constants $s_{i}, \theta_{j}$ such that

$$
\begin{gathered}
s_{i} a_{i i}^{\iota}>\sum_{k=1, k \neq i}^{N} s_{k} a_{k i}^{\mu}+\sum_{l=1}^{M} \theta_{l} c_{l i}^{\mu}, \\
\theta_{j} e_{j j}^{\iota}>\sum_{k=1}^{N} s_{k} d_{k j}^{\mu}+\sum_{l=1, l \neq j}^{M} \theta_{l} e_{l j}^{\mu},
\end{gathered}
$$

then system (E) has a unique almost-periodic solution $Q(t)$ in $\Omega(E)$.

Proof. Let $U \in S(E)$. First of all, we shall prove that $U$ is RTS for system $(E)$. By Lemma 3.6, it suffices to show that $U$ is RWUAS in $\Omega(E)$. For arbitrary $(\bar{U}, \bar{E}) \in \Omega(U, E)$ and $\bar{W} \in S(\bar{E})$, let

$$
\begin{aligned}
& V(t)=V(t, \bar{U}(\cdot), \bar{W}(\cdot)) \\
& =\sum_{i=1}^{N} s_{i}\left[\left|\ln \bar{u}_{i}(t)-\ln \bar{w}_{i}(t)\right|\right. \\
& \quad+\sum_{k=1, k \neq i}^{N} \int_{0}^{\infty} H_{i k}(s)\left\{\int_{t-s}^{t} \bar{a}_{i k}(s+\sigma)\left|\bar{u}_{k}(\sigma)-\bar{w}_{k}(\sigma)\right| \mathrm{d} \sigma\right\} \mathrm{d} s \\
& \left.\quad+\sum_{l=1}^{M} \int_{0}^{\infty} K_{i l}(s)\left\{\int_{t-s}^{t} \bar{c}_{i l}(s+\sigma)\left|\bar{v}_{l}(\sigma)-\bar{z}_{l}(\sigma)\right| \mathrm{d} \sigma\right\} \mathrm{d} s\right]
\end{aligned}
$$




$$
\begin{aligned}
&+\sum_{j=1}^{M} \theta_{j}\left[\left|\ln \bar{v}_{j}(t)-\ln \bar{z}_{j}(t)\right|\right. \\
&+\sum_{k=1}^{N} \int_{0}^{\infty} P_{j k}(s)\left\{\int_{t-s}^{t} \bar{d}_{j k}(s+\sigma)\left|\bar{u}_{k}(\sigma)-\bar{w}_{k}(\sigma)\right| \mathrm{d} \sigma\right\} \mathrm{d} s \\
&\left.\quad+\sum_{l=1, l \neq j}^{M} \int_{0}^{\infty} Q_{j l}(s)\left\{\int_{t-s}^{t} \bar{e}_{j l}(s+\sigma)\left|\bar{v}_{l}(\sigma)-\bar{z}_{l}(\sigma)\right| \mathrm{d} \sigma\right\} \mathrm{d} s\right] .
\end{aligned}
$$

We denote

$$
\lambda=\min _{i, j}\left\{s_{i}, \theta_{j}\right\}, \quad \Lambda=\max _{i, j}\left\{s_{i}, \theta_{j}\right\} .
$$

Calculating the right derivative $D^{+} V(t)$ of $V$ along system $(E)-(2.1)$, we obtain

$$
\begin{aligned}
& D^{+} V(t)=\sum_{i=1}^{N} s_{i}\left\{\operatorname{sgn}\left\{\bar{u}_{i}(t)-\bar{w}_{i}(t)\right\}\right. \\
& \times\left[-\bar{a}_{i i}(t)\left(\bar{u}_{i}(t)-\bar{w}_{i}(t)\right)\right. \\
& -\sum_{k=1, k \neq i}^{N} \bar{a}_{i k}(t) \int_{-\infty}^{t} H_{i k}(t-\sigma)\left(\bar{u}_{k}(\sigma)-\bar{w}_{k}(\sigma)\right) \mathrm{d} \sigma \\
& -\sum_{l=1}^{M} \bar{c}_{i l}(t) \int_{-\infty}^{t} K_{i l}(t-\sigma)\left(\bar{v}_{l}(\sigma)-\bar{z}_{l}(\sigma)\right) \mathrm{d} \sigma \\
& +\sum_{k=1, k \neq i}^{N} \int_{0}^{\infty} H_{i k}(s) \bar{a}_{i k}(s+t) \mathrm{d} s\left|\bar{u}_{k}(t)-\bar{w}_{k}(t)\right| \\
& +\sum_{k=1, k \neq i}^{N} \bar{a}_{i k}(t) \int_{-\infty}^{t} H_{i k}(t-\sigma)\left|\bar{u}_{k}(\sigma)-\bar{w}_{k}(\sigma)\right| \mathrm{d} \sigma \\
& +\sum_{l=1}^{M} \int_{0}^{\infty} K_{i l}(s) \bar{c}_{i l}(s+t) \mathrm{d} s\left|\bar{v}_{l}(t)-\bar{z}_{l}(t)\right| \\
& \left.\left.+\sum_{l=1}^{M} \bar{c}_{i l}(t) \int_{-\infty}^{t} K_{i l}(t-\sigma)\left|\bar{v}_{l}(\sigma)-\bar{z}_{l}(\sigma)\right| \mathrm{d} \sigma\right]\right\} \\
& +\sum_{j=1}^{M} \theta_{j}\left\{\operatorname{sgn}\left\{\bar{v}_{j}(t)-\bar{z}_{j}(t)\right\}\right. \\
& \times\left[-\bar{e}_{j j}(t)\left(\bar{v}_{j}(t)-\bar{z}_{j}(t)\right)\right. \\
& +\sum_{k=1}^{N} \bar{d}_{j k}(t) \int_{-\infty}^{t} P_{j k}(t-\sigma)\left(\bar{u}_{k}(\sigma)-\bar{w}_{k}(\sigma)\right) \mathrm{d} \sigma
\end{aligned}
$$




$$
\begin{aligned}
& -\sum_{l=1, l \neq j}^{M} \bar{e}_{j l}(t) \int_{-\infty}^{t} Q_{j l}(t-\sigma)\left(\bar{v}_{l}(\sigma)-\bar{z}_{l}(\sigma)\right) \mathrm{d} \sigma \\
& +\sum_{k=1}^{N} \int_{0}^{\infty} P_{j k}(s) \bar{d}_{j k}(s+t) \mathrm{d} s\left|\bar{u}_{k}(t)-\bar{w}_{k}(t)\right| \\
& -\sum_{k=1}^{N} \bar{d}_{j k}(t) \int_{-\infty}^{t} P_{j k}(t-\sigma)\left|\bar{u}_{k}(\sigma)-\bar{w}_{k}(\sigma)\right| \mathrm{d} \sigma \\
& +\sum_{l=1, l \neq j}^{M} \int_{0}^{\infty} Q_{j l}(s) \bar{e}_{j l}(s+t)\left|\bar{v}_{l}(t)-\bar{z}_{l}(t)\right| \\
& \left.\left.\quad+\sum_{l=1, l \neq j}^{M} \bar{e}_{j l}(t) \int_{-\infty}^{t} Q_{j l}(t-\sigma)\left|\bar{v}_{l}(\sigma)-\bar{z}_{l}(\sigma)\right| \mathrm{d} \sigma\right]\right\}
\end{aligned}
$$

By simplifying, one obtains

$$
\begin{aligned}
D^{+} V(t) \leqslant & \sum_{i=1}^{N}\left[\sum_{k=1, k \neq i}^{N} s_{k} a_{k i}^{\mu}+\sum_{l=1}^{M} \theta_{l} c_{l i}^{\mu}-s_{i} a_{i i}^{\iota}\right]\left|\bar{u}_{i}(t)-\bar{w}_{i}(t)\right| \\
& +\sum_{j=1}^{M}\left[\sum_{k=1}^{N} s_{k} d_{k j}^{\mu}+\sum_{l=1, l \neq j}^{M} \theta_{l} e_{l j}^{\mu}-\theta_{j} e_{j j}^{\iota}\right]\left|\bar{v}_{j}(t)-\bar{z}_{j}(t)\right| \\
\leqslant & -\gamma\left[\sum_{i=1}^{N}\left|\bar{u}_{i}(t)-\bar{w}_{i}(t)\right|+\sum_{j=1}^{M}\left|\bar{v}_{j}(t)-\bar{z}_{j}(t)\right|\right]
\end{aligned}
$$

where

$$
\gamma=\min _{i, j}\left\{s_{i} a_{i i}^{\iota}-\sum_{k=1, k \neq i}^{N} s_{k} a_{k i}^{\mu}-\sum_{l=1}^{M} \theta_{l} c_{l i}^{\mu}, \theta_{j} e_{j j}^{\iota}-\sum_{k=1}^{N} s_{k} d_{k j}^{\mu}-\sum_{l=1, l \neq j}^{M} \theta_{l} e_{l j}^{\mu}\right\} .
$$

From $\left(\mathrm{H}_{4}\right)$, we know that $\gamma>0$. Integrating (3.5) over $[0, t]$, it follows that

$$
V(t)+\gamma \int_{0}^{t}\left[\sum_{i=1}^{N}\left|\bar{u}_{i}(s)-\bar{w}_{i}(s)\right|+\sum_{j=1}^{M}\left|\bar{v}_{j}(s)-\bar{z}_{j}(s)\right|\right] \mathrm{d} s<V(0)<+\infty \quad \text { for } t \geqslant 0 .
$$

Consequently,

$$
\int_{0}^{t}\left[\sum_{i=1}^{N}\left|\bar{u}_{i}(s)-\bar{w}_{i}(s)\right|+\sum_{j=1}^{M}\left|\bar{v}_{j}(s)-\bar{z}_{j}(s)\right|\right] \mathrm{d} s<\infty .
$$

Since the function

$$
\sum_{i=1}^{N}\left|\bar{u}_{i}(t)-\bar{w}_{i}(t)\right|+\sum_{j=1}^{M}\left|\bar{v}_{j}(t)-\bar{z}_{j}(t)\right|
$$


is uniformly continuous on $[0,+\infty)$, we have

$$
\sum_{i=1}^{N}\left|\bar{u}_{i}(t)-\bar{w}_{i}(t)\right|+\sum_{j=1}^{M}\left|\bar{v}_{j}(t)-\bar{z}_{j}(t)\right| \rightarrow 0,
$$

and thus $\rho\left(\bar{U}^{t}, \bar{W}^{t}\right) \rightarrow 0$ as $t \rightarrow \infty$. Moreover, from (3.4) and (3.6), it follows that

$$
\begin{aligned}
& \sum_{i=1}^{N}\left|\ln \bar{u}_{i}(t)-\ln \bar{w}_{i}(t)\right|+\sum_{j=1}^{M}\left|\ln \bar{v}_{j}(t)-\ln \bar{z}_{j}(t)\right| \\
& \leqslant \frac{V(t)}{\lambda} \leqslant \frac{V\left(t_{0}\right)}{\lambda} \\
& \leqslant \frac{2 x^{*} \Lambda}{\lambda} \sum_{i=1}^{n}\left[\sum_{k=1, k \neq i}^{N} a_{i k}^{\mu} \int_{L}^{\infty} s H_{i k}(s) \mathrm{d} s+\sum_{l=1}^{M} c_{i l}^{\mu} \int_{L}^{\infty} s K_{i l}(s) \mathrm{d} s\right] \\
& +\frac{2 x^{*} \Lambda}{\lambda} \sum_{j=1}^{M}\left[\sum_{k=1}^{N} d_{j k}^{\mu} \int_{L}^{\infty} s P_{j k}(s) \mathrm{d} s+\sum_{l=1, l \neq j}^{M} e_{j l}^{\mu} \int_{L}^{\infty} s Q_{j l}(s) \mathrm{d} s\right] \\
& +\frac{1}{\lambda} \sum_{i=1}^{N} s_{i}\left\{\left|\ln \bar{u}_{i}\left(t_{0}\right)-\ln \bar{w}_{i}\left(t_{0}\right)\right|\right. \\
& +\sum_{k=1, k \neq i}^{N} a_{i k}^{\mu} \int_{0}^{\infty} s H_{i k}(s) \mathrm{d} s \sup _{t_{0}-L \leqslant \sigma \leqslant t_{0}}\left|\bar{u}_{k}(\sigma)-\bar{w}_{k}(\sigma)\right| \\
& \left.+\sum_{l=1}^{M} c_{i l}^{\mu} \int_{0}^{\infty} s K_{i l}(s) \mathrm{d} s \sup _{t_{0}-L \leqslant \sigma \leqslant t_{0}}\left|\bar{v}_{l}(\sigma)-\bar{z}_{l}(\sigma)\right|\right\} \\
& +\frac{1}{\lambda} \sum_{j=1}^{M} \theta_{j}\left\{\left|\ln \bar{v}_{j}\left(t_{0}\right)-\ln \bar{z}_{j}\left(t_{0}\right)\right|\right. \\
& +\sum_{k=1}^{N} d_{j k}^{\mu} \int_{0}^{\infty} s P_{j k}(s) \mathrm{d} s \sup _{t_{0}-L \leqslant \sigma \leqslant t_{0}}\left|\bar{u}_{k}(\sigma)-\bar{w}_{k}(\sigma)\right| \\
& \left.+\sum_{l=1, l \neq j}^{M} e_{j l}^{\mu} \int_{0}^{\infty} s Q_{j l}(s) \mathrm{d} s \sup _{t_{0}-L \leqslant \sigma \leqslant t_{0}}\left|\bar{v}_{l}(\sigma)-\bar{z}_{l}(\sigma)\right|\right\} .
\end{aligned}
$$

for all $t \geqslant t_{0} \geqslant 0$, and all $L \geqslant 0$. For each $\varepsilon>0$ we set

$$
\begin{array}{r}
\bar{\delta}(\varepsilon)=\inf \left\{\sum_{i=1}^{N}\left|\ln u_{i}(t)-\ln w_{i}(t)\right|+\sum_{j=1}^{M}\left|\ln v_{j}(t)-\ln z_{j}(t)\right|:|U-W| \geqslant \varepsilon\right. \\
\text { and } \left.\alpha_{i} \leqslant u_{i}(t), w_{i}(t) \leqslant p_{i}, \beta_{j} \leqslant v_{j}(t), z_{j}(t) \leqslant q_{j}\right\} .
\end{array}
$$


Obviously, $\bar{\delta}(\varepsilon)>0$. We select a number $L>0$ so large that

$$
\begin{aligned}
\left\{2 x^{*} \Lambda \sum_{i=1}^{N}\right. & {\left[\sum_{k=1, k \neq i}^{N} a_{i k}^{\mu} \int_{L}^{\infty} s H_{i k}(s) \mathrm{d} s+\sum_{l=1}^{M} c_{i l}^{\mu} \int_{L}^{\infty} s K_{i l}(s) \mathrm{d} s\right] } \\
& \left.+2 x^{*} \Lambda \sum_{j=1}^{M}\left[\sum_{k=1}^{N} d_{j k}^{\mu} \int_{L}^{\infty} s P_{j k}(s) \mathrm{d} s+\sum_{l=1, l \neq j}^{M} e_{j l}^{\mu} \int_{L}^{\infty} s Q_{j l}(s) \mathrm{d} s\right]\right\}<\frac{1}{2} \lambda \bar{\delta}(\varepsilon),
\end{aligned}
$$

which is possible by $\left(\mathrm{H}_{2}\right)$. Moreover, we select a $\delta(\varepsilon) \in(0, \varepsilon)$ so that

$$
\begin{aligned}
\sum_{i=1}^{N} s_{i}\left[\left|\ln \phi_{i}(0)-\ln \xi_{i}(0)\right|\right. & +\sum_{k=1, k \neq i}^{N} a_{i k}^{\mu} \int_{0}^{\infty} s H_{i k}(s) \mathrm{d} s \sup _{t_{0}-L \leqslant \sigma \leqslant t_{0}}\left|\phi_{k}(\sigma)-\xi_{k}(\sigma)\right| \\
& \left.+\sum_{l=1}^{M} c_{i l}^{\mu} \int_{0}^{\infty} s K_{i l}(s) \mathrm{d} s \sup _{t_{0}-L \leqslant \sigma \leqslant t_{0}}\left|\psi_{l}(\sigma)-\eta_{l}(\sigma)\right|\right] \\
+\sum_{j=1}^{M} \theta_{j}\left[\left|\ln \psi_{j}(0)-\ln \eta_{j}(0)\right|\right. & +\sum_{k=1}^{N} d_{j k}^{\mu} \int_{0}^{\infty} s P_{j k}(s) \mathrm{d} s \sup _{t_{0}-L \leqslant \sigma \leqslant t_{0}}\left|\phi_{k}(\sigma)-\xi_{k}(\sigma)\right| \\
& \left.+\sum_{l=1, l \neq j}^{M} e_{j l}^{\mu} \int_{0}^{\infty} s Q_{j l}(s) \mathrm{d} s \sup _{t_{0}-L \leqslant \sigma \leqslant t_{0}}\left|\psi_{l}(\sigma)-\eta_{l}(\sigma)\right|\right]<\frac{1}{2} \lambda \bar{\delta}(\varepsilon),
\end{aligned}
$$

whenever $\rho(\Phi, \Psi)<\delta(\varepsilon)$. Hence, if $\rho\left(\bar{U}^{t_{0}}, \bar{W}^{t_{0}}\right)<\delta(\varepsilon)$, we have

$$
\sum_{i=1}^{N}\left|\ln \bar{u}_{i}(t)-\ln \bar{w}_{i}(t)\right|+\sum_{j=1}^{M}\left|\ln \bar{v}_{j}(t)-\ln \bar{z}_{j}(t)\right|<\bar{\delta}(\varepsilon) .
$$

By (3.7) and (3.8), consequently, $|\bar{U}(t)-\bar{W}(t)|<\varepsilon$ for all $t \geqslant t_{0}$. Thus, if $\rho\left(\bar{U}^{t_{0}}, \bar{W}^{t_{0}}\right)<$ $\delta(\varepsilon)$, then

$$
\begin{aligned}
\rho\left(\bar{U}^{t}, \bar{W}^{t}\right) & \leqslant \sum_{n=1}^{\infty} \frac{\rho_{n}\left(\bar{U}^{t_{0}}, \bar{W}^{t_{0}}\right)+\varepsilon}{2^{n}\left(1+\rho_{n}\left(\bar{U}^{t_{0}}, \bar{W}^{t_{0}}\right)+\varepsilon\right.} \\
& \leqslant \sum_{n=1}^{\infty} 2^{-n} \frac{\rho_{n}\left(\bar{U}^{t_{0}}, \bar{W}^{t_{0}}\right)}{\left(1+\rho_{n}\left(\bar{U}^{t_{0}}, \bar{W}^{t_{0}}\right)+(\varepsilon /(1+\varepsilon))\right.} \\
& \leqslant \delta(\varepsilon)+\varepsilon \\
& <2 \varepsilon,
\end{aligned}
$$

for all $t \geqslant t_{0}$. Note that $\delta(\cdot)$ is independent of the particular choice of $\bar{U}, \bar{W} \in S(\bar{E})$. Therefore, each $U \in S(E)$ is RWUAS in $\Omega(E)$.

Next, we shall prove that each $U \in S(E)$ is asymptotically almost periodic. Let $\left\{t_{n}\right\}$ be any sequence satisfying $t_{n} \rightarrow \infty$ as $n \rightarrow \infty$. We may assume that the sequence $\left\{U\left(t+t_{n}\right)\right\}_{n=1}^{\infty}$ is uniformly convergent on each bounded subset of $\mathbb{R}$ and that the 
sequences

$$
\begin{array}{lll}
\left\{b_{i}\left(t+t_{n}\right)\right\}_{n=1}^{\infty}, & \left\{r_{j}\left(t+t_{n}\right)\right\}_{n=1}^{\infty}, & \left\{a_{i k}\left(t+t_{n}\right)\right\}_{n=1}^{\infty}, \\
\left\{c_{i l}\left(t+t_{n}\right)\right\}_{n=1}^{\infty}, & \left\{d_{j k}\left(t+t_{n}\right)\right\}_{n=1}^{\infty}, & \left\{e_{j l}\left(t+t_{n}\right)\right\}_{n=1}^{\infty}
\end{array}
$$

are uniformly convergent on $\mathbb{R}$. Set $U^{m}(t)=U\left(t+t_{m}\right), t \in \mathbb{R}$, for each positive integer $m$. Clearly, $U^{m}$ is a solution of the system

$$
\left.\begin{array}{rl}
\dot{x}_{i}(t)=x_{i}(t)[ & b_{i}\left(t+t_{m}\right)-a_{i i}\left(t+t_{m}\right) x_{i}(t)-\sum_{k=1, k \neq i}^{N} a_{i k}\left(t+t_{m}\right) \\
& \left.\times \int_{-\infty}^{t} H_{i k}(t-\sigma) x_{k}(\sigma) \mathrm{d} \sigma-\sum_{l=1}^{M} c_{i l}\left(t+t_{m}\right) \int_{-\infty}^{t} K_{i l}(t-\sigma) y_{l}(\sigma) \mathrm{d} \sigma\right], \\
\dot{y}_{j}(t)=y_{j}(t)[ & -r_{j}\left(t+t_{m}\right)-e_{j j}\left(t+t_{m}\right) y_{j}(t)+\sum_{k=1}^{N} d_{j k}\left(t+t_{m}\right) \\
& \left.\times \int_{-\infty}^{t} P_{j k}(t-\sigma) x_{k}(\sigma) \mathrm{d} \sigma-\sum_{l=1, l \neq j}^{M} e_{j l}\left(t+t_{m}\right) \int_{-\infty}^{t} Q_{j l}(t-\sigma) y_{l}(\sigma) \mathrm{d} \sigma\right]
\end{array}\right\}
$$

on $\mathbb{R}$ and it is RTS for system $\left(E^{m}\right)$ with the common number $\delta(\cdot)$, since $U$ is RTS for system $(E)$ with the common number $\delta(\cdot)$. For any positive integers $m$ and $n$, we define a continuous function $G_{m n}: R \rightarrow R^{N+M}$ by

$$
G_{m n}(t)=\left(f_{m n 1}(t), \ldots, f_{m n N}(t), g_{m n 1}(t), \ldots, g_{m n M}(t)\right)
$$

where

$$
\begin{aligned}
f_{m n i}(t)=u_{i}\left(t+t_{n}\right)[ & b_{i}\left(t+t_{n}\right)-b_{i}\left(t+t_{m}\right)-\left(a_{i i}\left(t+t_{n}\right)-a_{i i}\left(t+t_{m}\right)\right) u_{i}\left(t+t_{n}\right) \\
& -\sum_{k=1, k \neq i}^{N}\left(a_{i k}\left(t+t_{n}\right)-a_{i k}\left(t+t_{m}\right)\right) \int_{-\infty}^{t} H_{i k}(t-\sigma) u_{k}\left(\sigma+t_{n}\right) \mathrm{d} \sigma \\
& \left.-\sum_{l=1}^{M}\left(c_{i l}\left(t+t_{n}\right)-c_{i l}\left(t+t_{m}\right)\right) \int_{-\infty}^{t} K_{i l}(t-\sigma) v_{l}\left(\sigma+t_{n}\right) \mathrm{d} \sigma\right], \\
g_{m n j}(t)=v_{j}\left(t+t_{n}\right)\left[-\left(r_{j}\left(t+t_{n}\right)-r_{j}\left(t+t_{m}\right)\right)-\left(e_{j j}\left(t+t_{n}\right)-e_{j j}\left(t+t_{m}\right)\right) v_{j}\left(t+t_{n}\right)\right. & \\
& +\sum_{k=1}^{N}\left(d_{j k}\left(t+t_{n}\right)-d_{j k}\left(t+t_{m}\right)\right) \int_{-\infty}^{t} P_{j k}(t-\sigma) u_{k}\left(\sigma+t_{n}\right) \mathrm{d} \sigma \\
& \left.-\sum_{l=1, l \neq j}^{M}\left(e_{j l}\left(t+t_{n}\right)-e_{j l}\left(t+t_{m}\right)\right) \int_{-\infty}^{t} Q_{j l}(t-\sigma) v_{l}\left(\sigma+t_{n}\right) \mathrm{d} \sigma\right] .
\end{aligned}
$$

Now, for any $\varepsilon>0$ there exists a positive integer $n_{0}(\varepsilon)$ such that $\sup _{t \in R}\left|G_{m n}(t)\right|<$ $\delta(\varepsilon)$ and $\rho\left(\left(U^{m}\right)^{0},\left(U^{n}\right)^{0}\right)<\delta(\varepsilon)$ if $m, n \geqslant n_{0}(\varepsilon)$. Then, the fact that $U^{m}$ is RTS for 
system $\left(E_{m}\right)$ implies that $\rho\left(\left(U^{m}\right)^{t},\left(U^{n}\right)^{t}\right)<\varepsilon$ for all $t \geqslant 0$ if $m, n \geqslant n_{0}(\varepsilon)$, since $U^{m}$ is a solution of system $\left(E_{G_{m n}}^{m}\right)$ on $\mathbb{R}$ and $\alpha_{i} \leqslant\left(u_{i}^{n}\right)(t) \leqslant p_{i}, \beta_{j} \leqslant\left(v_{j}\right)^{n}(t) \leqslant q_{j}$ for all $t \in \mathbb{R}$. Thus, the sequence $\left\{U\left(t+t_{n}\right)\right\}_{n=1}^{\infty}$ is uniformly convergent on $[0, \infty)$, which shows that $U(t)$ is asymptotically almost-periodic, by Lemma 3.7, that is, $U(t)$ is the sum of an almost-periodic function $Q(t)$ and a continuous function $\gamma(t)$ defined on $\mathbb{R}$ such that $U(t)=Q(t)+\gamma(t), t \in \mathbb{R}$, and $\gamma(t) \rightarrow 0$ as $t \rightarrow \infty$.

Finally, we show that $Q$ is a unique almost-periodic solution in $S(E)$. We choose a sequence $\left\{s_{n}\right\}, s_{n} \rightarrow \infty$, as $n \rightarrow \infty$ such that

$$
\begin{gathered}
b_{i}\left(t+s_{n}\right) \rightarrow b_{i}(t), \quad r_{j}\left(t+s_{n}\right) \rightarrow r_{j}(t), \quad a_{i k}\left(t+s_{n}\right) \rightarrow a_{i k}(t), \quad c_{i l}\left(t+s_{n}\right) \rightarrow c_{i l}(t), \\
d_{j k}\left(t+s_{n}\right) \rightarrow d_{j k}(t), \quad e_{j l}\left(t+s_{n}\right) \rightarrow e_{j l}(t), \quad Q\left(t+s_{n}\right) \rightarrow Q(t),
\end{gathered}
$$

as $n \rightarrow \infty$ uniformly on $\mathbb{R}$. Then, $Q \in S(E)$ by Theorem 2.4. Let $\bar{Q}$ be another almostperiodic solution in $S(E)$. Since $Q \in S(E)$ is RWUAS in $\Omega(E)$, as was shown in the first paragraph of the proof of the theorem, we obtain $\rho\left(Q^{t}, \bar{Q}^{t}\right) \rightarrow 0$ as $t \rightarrow \infty$ and hence $|Q(t)-\bar{Q}(t)| \rightarrow 0$ as $t \rightarrow \infty$. Hence, $Q \equiv \bar{Q}$ on $\mathbb{R}$, by the almost-periodicity of $Q$ and $\bar{Q}$. Thus, system $(E)$ has $Q$ as a unique almost-periodic solution in $S(E)$. This completes the proof of Theorem 3.8.

Corollary 3.9. Under the assumptions $\left(\mathrm{H}_{2}\right)-\left(\mathrm{H}_{4}\right)$ and supposing that $b_{i}, r_{j}, a_{i k}, c_{i l}$, $d_{j k}$ and $e_{j l}$ are all $\omega$-periodic, system $(E)$ then has a unique $\omega$-periodic solution in $S(E)$.

Proof. By Theorem 3.8, let $F$ be the unique positive almost-periodic solution of system $(E)$, but in the periodic case, $b_{i}, r_{j}, a_{i k}, c_{i l}, d_{j k}$ and $e_{j l}$ are all $\omega$-periodic. Therefore, $F(t+\omega)$ is also an almost-periodic solution of system $(E)$. By the uniqueness of almost-periodic solutions, it follows that $F(t)=F(t+\omega)$ for all $t \in \mathbb{R}$. This completes the proof of Corollary 3.9.

When $M=0$, system $(E)$ degenerates to the Lotka-Volterra competition system

$x_{i}(t)=x_{i}(t)\left[b_{i}(t)-a_{i i}(t) x_{i}(t)-\sum_{k=1, k \neq i}^{N} a_{i k}(t) \int_{-\infty}^{t} H_{i k}(t-\sigma) x_{k}(\sigma) \mathrm{d} \sigma\right], \quad i=1, \ldots, N$,

which was considered by Gopalsamy $[\mathbf{1 1}]$.

Corollary 3.10. If system (3.9) satisfies the conditions

(i) $b_{i}, a_{i k}$ are all periodic with $b_{i}^{\iota}>0$ and $a_{i i}^{\iota}>0$,

(ii) $\int_{0}^{\infty} H_{i k}(s) \mathrm{d} s=1, \int_{0}^{\infty} s H_{i k}(s) \mathrm{d} s<\infty, i \neq k$,

(iii) $b_{i}^{\iota}>\sum_{k=1, k \neq i}^{N} a_{i k}^{\mu}\left(\frac{b_{k}^{\iota}}{a_{k k}^{\mu}}\right)$, 
(iv) there exist strictly positive constants $s_{i}$ such that

$$
s_{i} \min _{t \in[0, \omega]} a_{i i}(t)>\sum_{k=1, k \neq i}^{N}\left(\max _{t \in[0, \omega]} s_{k} a_{k i}(t)\right),
$$

then system (3.9) has a unique $\omega$-periodic solution.

Remark 3.11. Gopalsamy [11] has studied system (3.9). It has been proved that if (i)-(iii) hold and

$$
\min _{t \in[0, \omega]} a_{i i}(t)>\sum_{k=1, k \neq i}^{N}\left(\max _{t \in[0, \omega]} a_{k i}(t)\right),
$$

then system (3.9) has a unique $\omega$-periodic solution. Obviously, the results in $[\mathbf{1 1}]$ are a special case of Corollary 3.10. In fact, we take $s_{i} \equiv 1$; the result in [11] follows. Therefore, this result generalizes and improves the main results in [11]. In particular, when $N=1$, $M=0$, we also generalize the results in $[\mathbf{1 9}]$.

\section{Some examples}

Example 4.1. We consider the following system:

$$
\begin{array}{r}
\dot{x}_{1}(t)=x_{1}(t)\left[5+\sin 2 t-2 x_{1}(t)-\frac{1}{2} \int_{-\infty}^{t} \exp \{-(t-u)\} x_{2}(u) \mathrm{d} u\right. \\
\left.-\frac{1}{4}(1+\sin \sqrt{3} t) \int_{-\infty}^{t} \exp \{-(t-u)\} y_{1}(u) \mathrm{d} u\right], \\
\dot{x}_{2}(t)=x_{2}(t)\left[5+\cos 2 t-\frac{1}{2} \int_{-\infty}^{t} \exp \{-(t-u)\} x_{1}(u) \mathrm{d} u-2 x_{2}(t)\right. \\
\left.-\frac{1}{4}(1+\sin \sqrt{3} t) \int_{-\infty}^{t} \exp \{-(t-u)\} y_{1}(u) \mathrm{d} u\right], \\
\dot{y}_{1}(t)=y_{1}(t)\left[-\frac{1+\sin 3 t}{20}+\frac{1}{2} \int_{-\infty}^{t} \exp \{-(t-u)\} x_{1}(u) \mathrm{d} u\right. \\
+\infty
\end{array}
$$

Corresponding to system $(E)$, we have $b_{1}(t)=5+\sin 2 t, b_{2}(t)=5+\cos 2 t, r_{1}(t)=$ $\frac{1}{20}(1+\sin 3 t), a_{11}(t)=a_{22}(t)=2, d_{11}(t)=d_{12}(t)=\frac{1}{2}, c_{11}(t)=c_{12}(t)=\frac{1}{4}(1+\sin \sqrt{3} t)$ and $e_{11}(t)=1$. It is easy to verify that our result applies to (4.1). In fact,

(i) $\alpha_{1}=\alpha_{2}=\frac{1}{2}>0, q_{1}=3>0, \beta_{1}=\frac{2}{5}>0$,

(ii) letting $s_{1}=s_{2}=1, \theta_{1}=\frac{3}{2}$, we can obtain

$$
\begin{aligned}
& s_{1} a_{11}^{\iota}=1 \times 2>s_{2} a_{21}^{\mu}+\theta_{1} d_{11}^{\mu}=1 \times \frac{1}{2}+\frac{3}{2} \times \frac{1}{2}, \\
& s_{2} a_{22}^{\iota}=1 \times 2>s_{1} a_{12}^{\mu}+\theta_{1} d_{12}^{\mu}=1 \times \frac{1}{2}+\frac{3}{2} \times \frac{1}{2}, \\
& \theta_{1} e_{11}^{\iota}=\frac{3}{2} \times 1>s_{1} c_{11}^{\mu}+s_{2} c_{12}^{\mu}=1 \times \frac{1}{2}+1 \times \frac{1}{2} .
\end{aligned}
$$


Thus, Theorem 3.8 applies, and shows that there is a unique almost-periodic solution of (4.1).

Example 4.2. Consider the following system:

$$
\left.\begin{array}{l}
\dot{x}_{1}(t)=x_{1}(t)\left[5+\sin t-2 x_{1}(t)-\frac{1}{8}(1+\sin t) \int_{-\infty}^{t} \exp \{-(t-u)\} x_{2}(u) \mathrm{d} u\right], \\
\dot{x}_{2}(t)=x_{2}(t)\left[9+\cos t-2 \int_{-\infty}^{t} \exp \{-(t-u)\} x_{1}(u) \mathrm{d} u-2 x_{2}(t)\right] .
\end{array}\right\}
$$

Corresponding to system $(E)$, we have $b_{1}(t)=5+\sin t, b_{2}(t)=9+\cos t, a_{11}(t)=a_{22}(t)=$ $2, a_{12}(t)=\frac{1}{8}(1+\sin t), a_{21}(t)=2$ and $H_{12}(t)=H_{21}(t)=\exp (-t)$.

Since $\inf _{t \in \mathbb{R}} a_{11}(t)=2=\sup _{t \in \mathbb{R}} a_{21}=2$, we cannot apply [11, Theorem 2.1] to system (4.2). However, it is easy to verify that our result applies to (4.2). In fact, putting $s_{1}=1$ and $s_{2}=\frac{1}{2}$ yields

$$
\begin{aligned}
& s_{1} a_{11}^{\iota}(t)=1 \times 2>s_{2} a_{21}^{\mu}(t)=\frac{1}{2} \times 2, \\
& s_{2} a_{22}^{\iota}(t)=\frac{1}{2} \times 2>s_{1} a_{12}^{\mu}(t)=1 \times \frac{1}{4} .
\end{aligned}
$$

Thus, from Corollary 3.10, the system (4.2) has a unique periodic solution.

Acknowledgements. The work described here was jointly supported by the National Natural Science Foundation of China under Grant no. 60574043, the Natural Science Foundation of Jiangsu Province, China, under Grant no. BK2006093, the Qing-Lan Engineering Project of Jiangsu Province, China, the Foundation of Developing Science and Technology of Fuzhou University under Grant no. 0030824594, the Foundation of Fujian Education Bureau, under Grant no. JB05048, and the Natural Science Foundation of Fujian Province, China.

The authors thank the referees and editor for their valuable suggestions and comments, which improved the presentation of this paper.

\section{References}

1. C. Alvarez and A. C. Lazer, An application of topological degree to the periodic competing species problem, J. Austral. Math. Soc. B 28 (1986), 202-219.

2. M. ARAKI AND M. Kondo, Stability and transient behavior of composite systems, IEEE Trans. Automat. Contr. 17 (1972), 537-541.

3. E. Beretta AND Y. KUANG, Convergence results in a well-known delayed predator-prey system, J. Math. Analysis Applic. 204 (1996), 840-853.

4. T. Burton and V. Hutson, Repellers in systems with infinite delay, J. Math. Analysis Applic. 37 (1989), 240-263.

5. L. Chen, Mathematical models and methods in ecology (Scientific Press, Beijing, 1988; in Chinese).

6. J. C. EILBECK AND J. Lopez-Gomez, On the periodic Lotka-Volterra competition model, J. Math. Analysis Applic. 210 (1997), 58-87.

7. A. M. Fink, Almost periodic differential equations, Lecture Notes in Mathematics, Volume 377 (Springer, 1974). 
8. H. I. FREEDMAN AND S. RUAN, Uniform persistence in functional differential equations, J. Diff. Eqns 115 (1995), 173-192.

9. K. Gopalsamy, Exchange of equilibria in two-species Lotka-Volterra competition models, J. Austral. Math. Soc. B 24 (1982), 160-170.

10. K. Gopalsamy, Global asymptotic stability in a periodic Lotka-Volterra system, J. Austral. Math. Soc. B 27 (1985), 66-72.

11. K. Gopalsamy, Dynamics of an almost periodic integro-differential equation, Tohoku Math. J. 37 (1985), 323-332.

12. J. K. HALE AND J. KATO, Phase space for retarded equations with infinite delay, Funkcial. Ekvac. 21 (1978), 11-41.

13. Y. Hino, S. Murakami And T. NAito, Functional differential equations with infinite delay, Lecture Notes in Mathematics, Volume 1473 (Springer, 1991).

14. P. Korman, Some new results on the periodic competition model, J. Math. Analysis Applic. 171 (1992), 131-138.

15. Y. KUANG, Delay differential equations with application in population dynamics (Academic Press, New York, 1993).

16. J. Lopez-Gomez, R. Ortega And A. Tineo, The periodic predator-prey Lotka-Volterra model, Adv. Diff. Eqns 1 (1996), 403-423.

17. Z. MA AND W. WANG, Asymptotic behavior of predator-prey system with time-dependent coefficients, Applic. Analysis 34 (1989), 79-90.

18. S. MurakAmi, Almost periodic solutions of a system of integro-differential equations, Tohoku Math. J. 39 (1987), 71-79.

19. G. SEIFERT, Almost periodic solutions delay differential equations with infinite delays, $J$. Diff. Eqns 41 (1981), 416-425.

20. Z. TEnG, On the positive almost periodic solutions of a class of Lotka-Volterra type systems with delays, J. Math. Analysis Applic. 249 (2000), 433-444.

21. X. WEN, Global attractivity of positive solution of multispecies ecological competitionpredator delay system, Acta Math. Sinica 45 (2002), 83-92.

22. Y. XIA AND J. CAO, Almost periodicity in an ecological model with $M$-predators and N-preys by 'pure-delay type' system, Nonlin. Dynam. 39 (2005), 275-304.

23. Y. Xia, F. Chen, A. Chen And J. CAO, Existence and global attractivity of an almost periodic ecological model, Appl. Math. Computat. 157 (2004), 449-475.

24. Y. XIA, J. CAO, H. Zhang AND F. CHEN, Almost periodic solutions of $n$-species competitive system with feedback controls, J. Math. Analysis Applic. 294 (2004), 503-522.

25. P. YANG AND R. XU, Global attractivity of the periodic Lotka-Volterra system, J. Math. Analysis Applic. 233 (1999), 221-232.

26. T. YoshizaWA, Stability theory for the existence of periodic solutions and almost-periodic solutions, Applied Mathematical Science, Volume 14 (Springer, 1975).

27. J. Zhao And W. Chen, Global asymptotic stability of a periodic ecological model, Appl. Math. Computat. 147 (2004), 881-892.

28. X. ZHAO, The qualitative analysis of $n$-species Lotka-Volterra periodic competition systems, Math. Comput. Modelling 15 (1991), 3-8. 